\title{
Emergent Magnetic Monopole and Dipole Screening by Proximity Effect With Noble Metal
}

\section{Fernando Martins}

Universidade Federal de Viçosa

\section{Teônis de Paiva}

Universidade Federal de Viçosa

\section{Daniel Duarte}

Universidade Federal de Viçosa

João Rodrigues

Instituto Federal de Educação Ciência e Tecnologia do Norte de Minas Gerais

\section{Lucas Mól}

Universidade Federal de Minas Gerais

\section{Jerome Borme}

International Iberian Nanotechnology Laboratory

\section{Paulo Freitas}

International Iberian Nanotechnology Laboratory

Clodoaldo de Araujo ( $\sim$ dearaujo@ufv.br)

Universidade Federal de Viçosa

\section{Research Article}

Keywords: magnetic monopole, dipole screening, noble metal, proximity effect

Posted Date: December 31st, 2020

DOI: https://doi.org/10.21203/rs.3.rs-136395/v1

License: (c) (i) This work is licensed under a Creative Commons Attribution 4.0 International License.

Read Full License 


\title{
Emergent magnetic monopole and dipole screening by proximity effect with noble metal
}

\author{
Fernando F. Martins ${ }^{1}$, Teônis S. Paiva ${ }^{1}$, Daniel G. Duarte ${ }^{1}$, João H. Rodrigues ${ }^{2}$, Lucas A. \\ S. Mól $^{3}$, Jerome Borme ${ }^{4}$, Paulo P. Freitas ${ }^{4}$, and Clodoaldo I. L. de Araujo ${ }^{1, *}$ \\ ${ }^{1}$ Laboratory of Spintronics and Nanomagnetism (LabSpiN), Departamento de Física, Universidade Federal de \\ Viçosa, 36570-000 - Viçosa - Minas Gerais, Brazil \\ ${ }^{2}$ Núcleo de Física, Instituto Federal de Minas Gerais - Campus Bambuí, 38900-000 Bambuí, Minas Gerais, Brazil \\ ${ }^{3}$ Laboratório de Simulação, Departamento de Física, ICEx, Universidade Federal de Minas Gerais, 31720-901 Belo \\ Horizonte, Minas Gerais, Brazil \\ ${ }^{4}$ INL-International Iberian Nanotechnology Laboratory, 4715-330, Braga, Portugal \\ *dearaujo@ufv.br
}

\begin{abstract}
In this work we present emergent screening of magnetic monopole and dipole by the presence of 20nm aluminum cover layer on square artificial spin ice $(A S I)$ systems. Our results were obtained in base of magnetic atomic force measurements, performed after external magnetic field steps application. We show that the evolution of magnetization and monopole population is affected by the aluminum presence and attribute that phenomena to the proximity effect, which is responsible for the magnetization vanish of the first atomic layers at the ferromagnetic interface. Using experimental values to estimate the decrease in the nanomagnetic dipole value used in an emergent excitation model and in the switching field distribution heterogeneity used in simulations, we observe a very good agreement among experimental and simulation results. The presented emergent screening could be used in new ASI geometries for thermodynamic activation or proposition of devices with selective magnetic monopole mobility.
\end{abstract}

\section{Introduction}

The quantization of electronic charge could just be explained so far by the existence of magnetic monopoles ${ }^{1}$. Although, its scarcity to be probabilistic detected ${ }^{2}$ and difficulty of creation in particle accelerators due to its predicted high mass ${ }^{3}$, still leaves the existence of such elementary particles unproven. In condensed-matter systems, magnetic monopoles were first reported as low energy emergent quasiparticles in ferromagnetic crystals ${ }^{4}$ and have been mostly observed in pyrochlore crystals, denominated natural spin ices by resembling geometric frustration and residual entropy of water ice ${ }^{5}$. In those crystals, at very low temperatures in the range of $0.6-2 \mathrm{~K}$, magnetic field was used to break symmetry and align the non energetic strings connecting monopoles, allowing its transport to generate magnetricity ${ }^{6}$. The original proposition ${ }^{7}$ and experimental observation at room temperature ${ }^{8}$ of magnetic monopole quasiparticles in square array of nanomagnets, inspired a plethora of alternative designs proposition presenting singular properties ${ }^{9-15}$. Despite the fact that easy sample nanofabrication brings very interesting implications for novel technological applications, traditional artificial spin ice (ASI) systems present two main obstacles to achieve that purpose. One is the high Curie temperature of conventional Permalloy nanomagnets ${ }^{16}$, that prevents large scale ground state achievement by thermal effects ${ }^{17,18}$ and the other is the energetic string connecting Nambu monopoles ${ }^{19,20}$ in bidimensional ASI, making them not free for magnetricity as in its natural counterpart. Those problems were circumvented so far by utilization of thinner nanomagnets, close to superparamagnetic regime, lowering Curie temperatures ${ }^{21,22}$, or utilization of low dimension in size arrays to achieve full ground state ${ }^{23}$. Both strategies could be fundamental to record and erase excitations in future technological applications. In order to surmount the strong connection between opposite monopoles in two dimensional $A S I$, systems close to degeneracy with vanishing string energy by lattice stretch have been proposed ${ }^{24-26}$. More recently, the theoretical prediction of monopole freedom ${ }^{27}$ in real degenerate systems of three dimensional $A S I^{28}$ was experimentally realized ${ }^{29}$ and a magnetic monopole plasma could be characterized ${ }^{30}$. All those advances together with possibilities of information record by specific nanomagnet manipulation, with support of external field applied just below the nanomagnetic coercivity ${ }^{31}$, opened a pave for technological applications such as logic gates ${ }^{32}$ or memristors ${ }^{33}$ for neuromorphic applications. In this work we aim to introduce an alternative way to manipulate monopole density and mobility in square ASI. Such manipulation is allowed by the presence of a noble metal layer on top of nanomagnets and is attributed here to the increase in proximity effect phenomena at the interface, due to the increase of charge carriers. Another interesting feature observed is the 
decrease in the system disorder due to attenuation of nanomagnets interface defects. We present experimental results obtained from direct MFM measurements and support our findings numerically using the emergent model developed by some of us ${ }^{34}$.

\section{Results and Discussions}

For the reversal process characterizations we have saturated nanomagnets magnetization in the $\mathrm{x}$-axis direction and performed steps of external magnetic field to the saturation in the opposite direction. Using the MFM images acquired after each field step, we were able to follow magnetization evolution and monopole density as a function of the external field. Ferromagnetic alignment of macro spins at a vertex give magnetization $M_{x}= \pm 1$ and zero charge, while antiferromagnetic alignment allow emergent charge $Q= \pm 2$ and null magnetization, as depicted in Figure 1a. In figure 1b we exemplify how experimental data was acquired. Measurements were performed in $50 \mu^{2}$ area comprising $12 \times 12$ vertices and the number of horizontal nanoislands pointing to left or right was counted as well as the number of emergent monopoles for each magnetic field step. In the figure, emergent monopoles are highlighted by red and blue squares according to its charge signal. The evolution of magnetization and monopole density as a function of the external magnetic field, performed in samples with and without aluminum interface, were systematically observed in several lattices and sample positions investigated are summarized by the results measured in Sq1 geometry presented in Figure 2. In the hysteresis curve of Figure 2a, it is possible to observe reasonable coercivity decrease and vanishing of steps related to magnetostatic traps from nanomagnets defects when aluminum layer is present. Similar results were reported using vibrating sample magnetometry technique for $A S I$ in contact with normal metal and superconductor ${ }^{35}$. Most pronounced effect can be noticed in the monopole density evolution curve of Figure 2b, with smaller
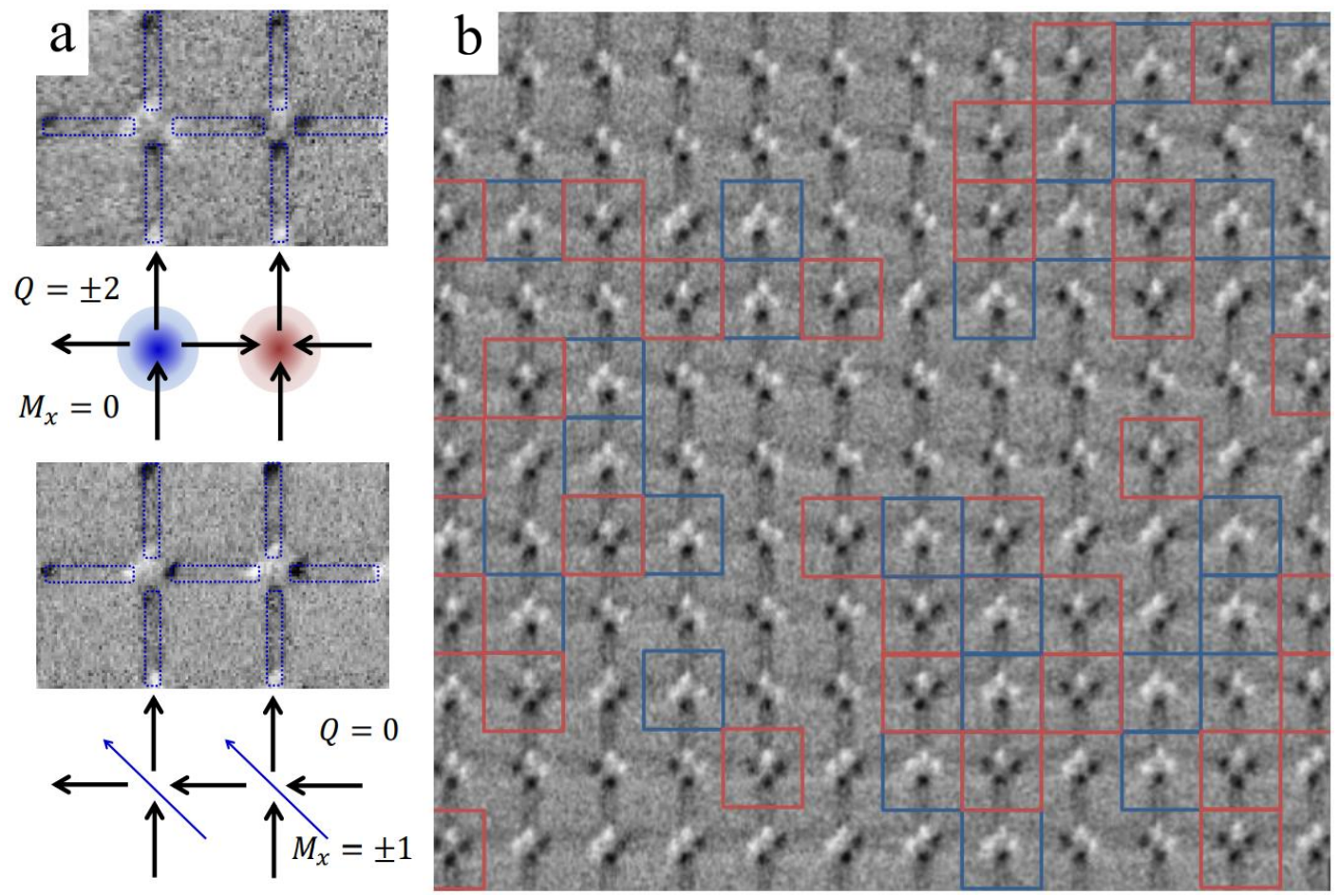

Figure 1. a) $M F M$ measurement at vertex showing respectively 3 out - 1 in / 3 in - 1 out configurations allowing emergence of charges $Q=+2$ and $Q=-2$, followed by 2 in - 2 out configuration of emergent dipole at vertex. b) Sample of measurement used to count magnetization and monopole density, realized during magnetization reversal process, with opposite charge monopoles highlighted by red and blue squares.

percentile of monopoles being generated in a sharp shape curve for lowest magnetic field. Those phenomena promoted by aluminum layer are qualitatively similar to what was observed in samples with different lattice parameters ${ }^{37}$ where it was found that increasing distances among nanomagnets at the vertex reduces the stray field from the nanomagnets, affecting directly the magnetic monopole and dipole intensity. The present aluminum layer effect suggest fascinating interaction among metallic electron gas with emergent magnetic monopole and dipole, decreasing its intensity in a sort of electric screening. In order to elucidate the origins of such physical phenomena, we call on experimental observations of spin polarization decay in first atomic layers of ferromagnetic thin films in contact with noble metals in spin valve structures ${ }^{38}$. Such magnetization suppression was theoretically investigated by first principle calculations and ascribed to hybridization of $s p-d$ orbitals at the interface ${ }^{36,39}$. 

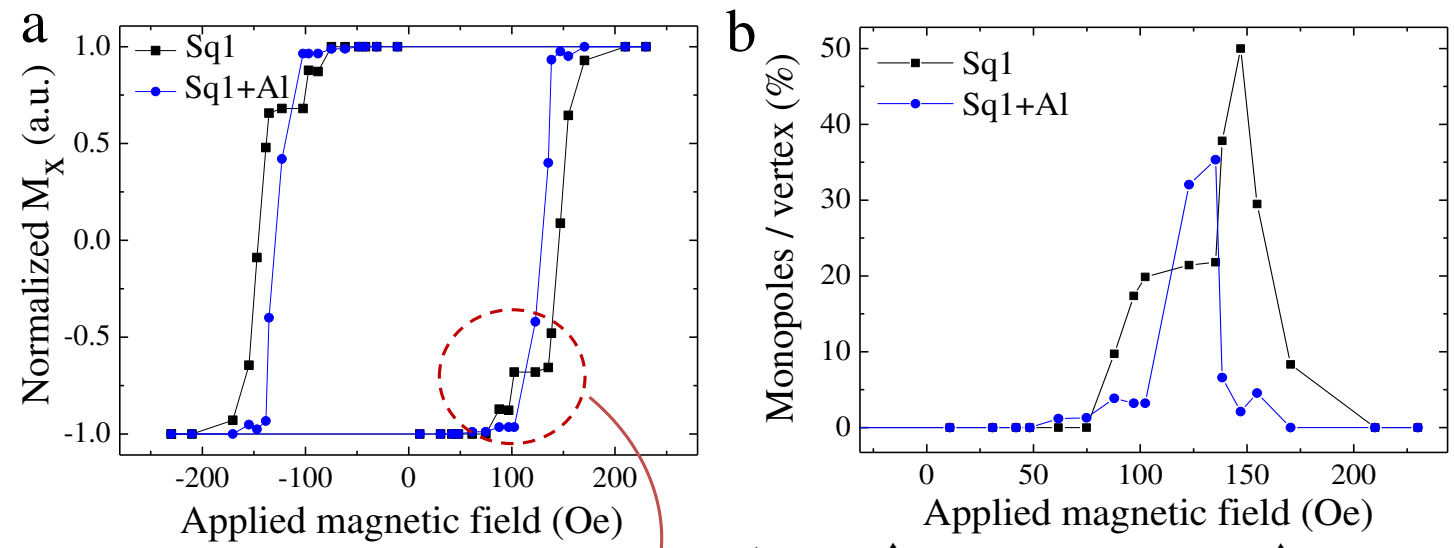

$\mathrm{C}$

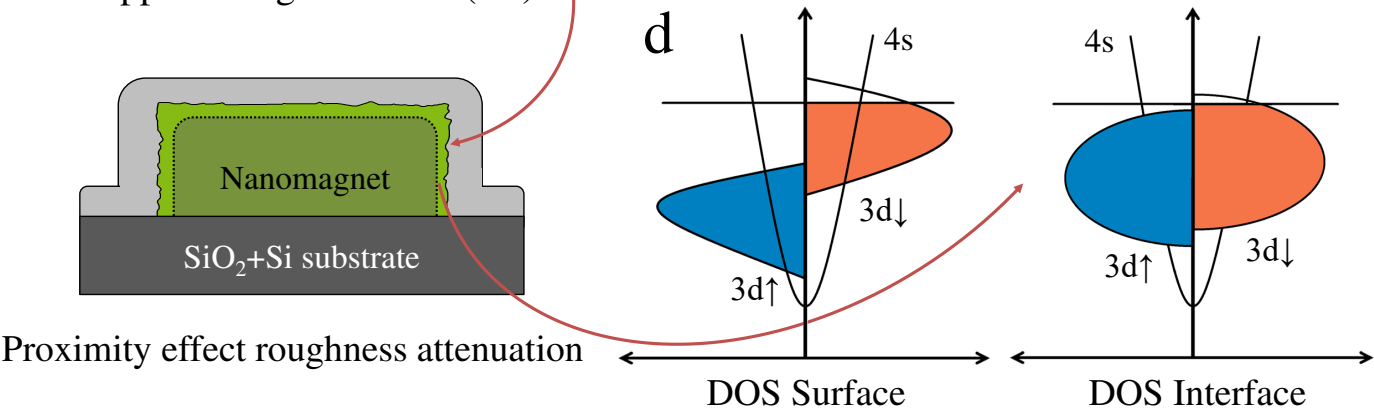

Figure 2. a) Hysteresis curve obtained by magnetization count at vertex during reversal process for Sq1 ASI sample and ASI samples covered by $20 \mathrm{~nm}$ aluminum $\mathrm{Sq} 1+\mathrm{Al}, \mathrm{b}$ ) Density of monopole evolution during reversal process, c) suggested defect smoothness by proximity effect and d) scheme of modification in density of state (DOS) by $s p-d$ hybridization at the interface ${ }^{36}$.

Scattering of electrons from $s p$ to $d$ orbital modify the density of states shape, decreasing spin density unbalance at Fermi level of ferromagnetic materials, as depicted in the cartoon of Figure 2c, extremely decreasing spin polarization of first atomic layers. Such interface magnetization suppression would also explain the vanishing of magnetostatic traps related to nanomagnets defects, once it would be smoothed by the proximity effect as suggested in Figure 2d. Emergent magnetic monopole charge and dipole intensity are totally related to the dipolar interaction, that in turn is related to the nanoisland's dipole moment. The increase in the proximity effect due to excess in charge carriers from noble metals would be responsible for intensity decrease of such emergent particles. To the best of our knowledge, experimental investigations or calculations of proximity effects by noble metals in nanostructures were not reported so far and in this work we are going to use an emergent excitation model ${ }^{34}$ to investigate how the magnetization interface decrease in nanomagnets due to charge carrier excess would imply in the observed behavior of magnetization and monopole density in the reversal processes of an ASI. In our previous work ${ }^{34,37}$, numerical solution of a model of emergent excitations that considers the presence of magnetic monopoles of charge $q$ and magnetic dipoles of moment $|\vec{M}|$ on the vertices, presented a qualitative match with the evolution of magnetization and monopole density in reversal process of experimental square $A S I$ arrays $^{37}$. In this model ${ }^{34}$, the total magnetic field at nanoisland $i, \vec{B}_{i}^{\text {tot }}$, is the sum of the field produced by the excitations on the vertices (see Refs. ${ }^{34,37}$ for details). added to the external magnetic field. The nanoisland magnetization (considered as a monodomain pointing in direction $\vec{S}_{i}$ ) is flipped when $\vec{B}_{i}^{t o t} \cdot \vec{S}_{i}<-h_{i}$, where $h_{i}$ is an intrinsic switching constant of nanoisland $i$. In order to take into account differences among nanoislands, the $h_{i}$ values are drawn from a Gaussian distribution centered at $h_{c}$ with standard deviation $\sigma_{d}=\sigma h_{c}$. In our previous work ${ }^{37}$, we found that in order to qualitatively describe experimental results, a kind of bimodal distribution had to be used, in such a way that for $90 \%$ of the nanoislands, we used $h_{c}=h_{c}^{90}$ and $\sigma^{90}$ and for the remaining $10 \%$ of the islands different values, $h_{c}=h_{c}^{10}$ and $\sigma^{10}$ were used. In general, $h_{c}^{x}$ will represent the mean switching field for $x \%$ of the nanoislands and $\sigma^{x} h_{c}^{x}$ the standard deviation of the gaussian distribution of switching fields for the same $x \%$ of the nanoislands. In the figure 3 experimental data for sample Sq2 is compared to simulations results. First, in figure 3a, we compare results for the sample without the aluminum cover. The outer plot shows part of the hysteresis curve and the inset shows the evolution of the magnetic monopoles density. The symbols represent the experimental data while solid curves are results from simulations averaged over 50 different distributions of switching fields. For the charge and dipole strengths we used the results we obtained in our previous work ${ }^{37}, q=0.5 \mu / a$ and $M=2.8 \mu$. The reversal field distribution was manually adjusted to fit the experimental curves. The best results were obtained 
a

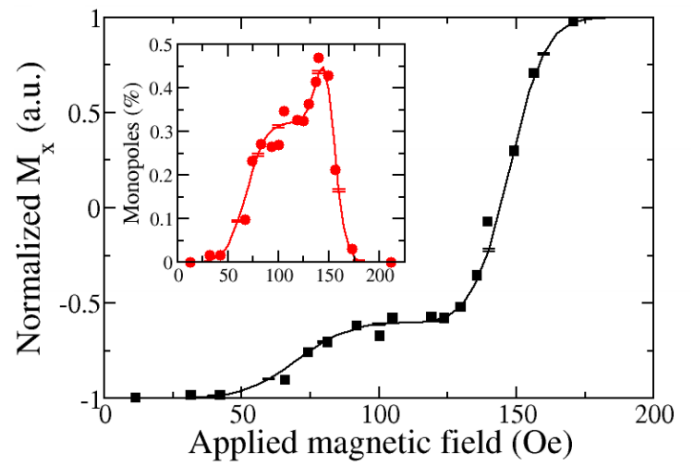

C

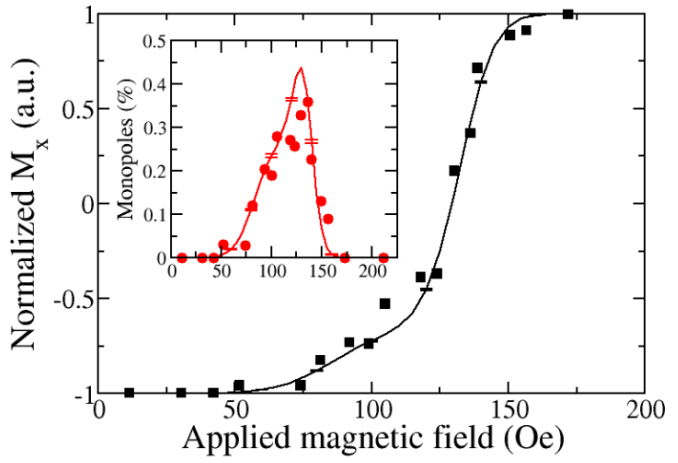

b

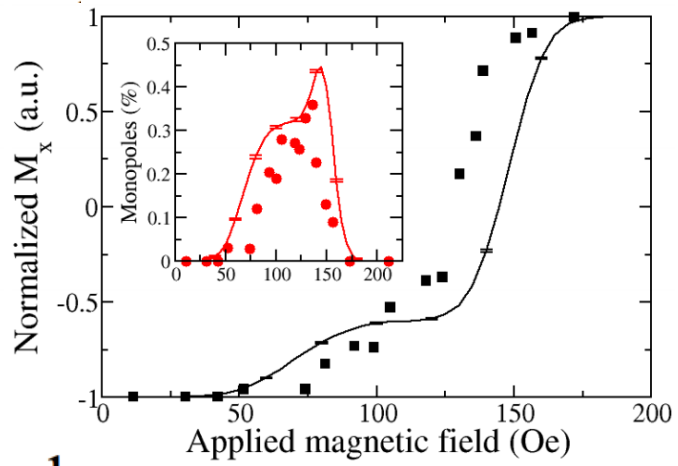

d

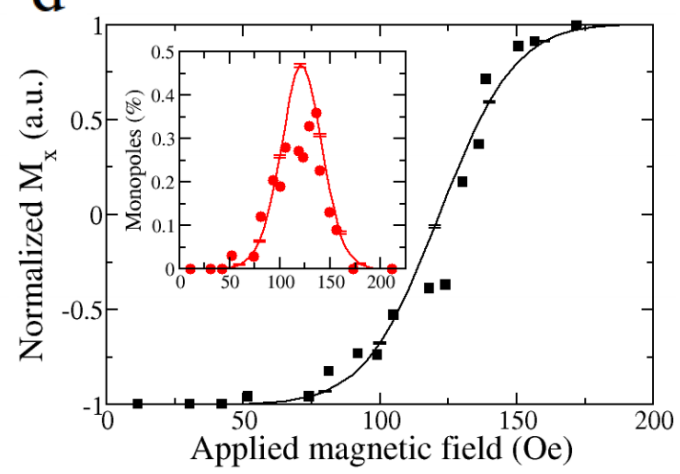

Figure 3. Experimental data for sample Sq2 (black squares and red dots) for the hysteresis (outer plots) and magnetic monopole density (insets) compared to results for simulations (solid curves). In a) we show the curves for the sample without the aluminum cover. Simulational results were obtained using $q=0.5 \mu / a, M=2.8 \mu, h_{c}^{80}=160 O e, \sigma^{80}=7.5 \%, h_{c}^{20}=80 O e$ and $\sigma^{20}=20 \%$. In b), c) and d) the curve for the sample with the aluminum cover is compared to simulations using different distributions for the reversal field of the nanoislands. In b) we used $q=0.475 \mu / a, M=2.71 \mu, h_{c}^{80}=1600 e, \sigma^{80}=7.5 \%$, $h_{c}^{20}=80 O e$ and $\sigma^{20}=20 \%$. In c) $q=0.475 \mu / a, M=2.71 \mu, h_{c}^{82}=144 O e, \sigma^{82}=8 \%, h_{c}^{18}=96 O e$ and $\sigma^{18}=10 \%$. In d) $q=0.475 \mu / a, M=2.71 \mu, h_{c}^{100}=133 O e, \sigma^{100}=18 \%$.

for $h_{c}^{80}=160 O e, \sigma^{80}=7.5 \%, h_{c}^{20}=80 O e$ and $\sigma^{20}=20 \%$. As can be seen, the agreement is remarkable! The aluminum cover is expected to cause interface effects, leading to polarization zero around $0.7 \mathrm{~nm}$ deep in nickel thin film ${ }^{38}$ (present in majority of Permalloy alloy used here). Thus, considering an effective thickness for the nanoislands of $19.3 \mathrm{~nm}$ instead of the original $20 \mathrm{~nm}$, the effective volume would be reduced by approximately $5 \%$, in such a way that a reduction of the same amount would be expected for the charge, $q$, and dipole moment, $M$, of emergent excitation. Figure $3 \mathrm{~b}$ shows the comparison among experimental results for the Sq2 sample with aluminum cover and simulational results considering $q=0.475 \mu / a, M=2.71 \mu$ and the same reversal fields used for the sample without the aluminum cover, $h_{c}^{80}=160 O e, \sigma^{80}=7.5 \%, h_{c}^{20}=80 O e$ and $\sigma^{20}=20 \%$. As can be seen, the results strongly suggest that the biggest effect of the aluminum cover will be on the switching fields and possibly on the reversal mechanism of the nanoislands. Interesting is the fact that the best adjust we achieved was obtained by changing the switching field parameters by about $10 \%$, as if they depend on the square of the volume. Figure $3 \mathrm{c}$ shows the comparison between experimental data and simulations performed using $q=0.475 \mu / a, M=2.71 \mu, h_{c}^{82}=144 O e$, $\sigma^{82}=8 \%, h_{c}^{18}=960 e$ and $\sigma^{18}=10 \%$. Now the bimodal distribution of switching fields approaches a unimodal distribution since the mean switching fields approaches each other. The heterogeneity present in the sample was reduced! Indeed, to give further support to this assertive, reasonable results were also obtained by considering a single Gaussian (unimodal) distribution centered at $h_{c}^{100}=133 \mathrm{Oe}$ with $\sigma^{100}=18 \%$ as can be seen in figure $3 \mathrm{~d}$, reinforcing the softening effect of the aluminum layer.

In summary we have investigated by direct $M F M$ measurements, performed during a magnetization reversal process of ASI samples, the evolution of emergent magnetic monopoles and dipoles in samples with and without the interface effect generated by $20 \mathrm{~nm}$ aluminum cover. Results show a systematic decrease in the coercivity and lowering of monopole creation density. The sharpening of the monopole density curve is related to a decrease in the heterogeneity of the distribution of the nanoislands switching fields and to a decrease on monopoles charge and dipoles moment. That decrease was attributed here to the annihilation of magnetostatic traps present on the nanomagnets surface by the magnetization vanishing in the first atomic 
layers of the nanomagnet interface and to the reduction of the overall magnetic moment of the nanoislands. Our simulation using an emergent model taking into account the value of nanomagnetic dipole under proximity effect supported our findings with very good accuracy. We believe that utilization of such covering may lead to more homogeneous systems, specially in what refers to the switching field of the nanoislands. In addition, our results suggest that the utilization of materials with controllable charge carrier density, in thinner nanomagnet samples, could be utilized for thermodynamic activation and utilization of partial covering in different geometries could be developed to allow investigations of samples with different monopole interaction and mobility.

\section{Methods}

We have performed our experimental observations in Permalloy square ASI samples, developed by sputtering deposition of Ta $3 \mathrm{~nm} / \mathrm{Py} 20 \mathrm{~nm} / \mathrm{Ta} 3 \mathrm{~nm}$ multi-layer, with Ta used both for adhesion and capping layer, with same geometry and thickness used in previous work ${ }^{37}$. After $M F M$ characterizations, samples were cleaned in acetone ultrasonic bath and $20 \mathrm{~nm}$ aluminum layer was deposited by thermal evaporation on top of the ASI geometries. Permalloy nanomagnets dimensions of $3 \mu \mathrm{m} \times 400 \mathrm{~nm} \times 20 \mathrm{~nm}$ are large enough to allow good magnetic force microscopy $(M F M)$ signal and sufficiently small to preserve the range where single magnetic monodomains are observed. Three different square lattice parameters were investigated with $M F M$ mapping realized in different regions of samples with and without aluminum cover. In all the measurements performed, aluminum capping effect was always observed. Here we focus on results obtained in Sample Sq1 with lattice parameter a $=3.95 \mu \mathrm{m}$ and $\mathrm{Sq} 2$ with $\mathrm{a}=4.35 \mu \mathrm{m}$, once that in those samples the presence of steps in hysteresis curve, attributed to sample defects originating cracking reversal ${ }^{37}$, and its attenuation by aluminum layers are more pronounced.

\section{References}

1. Dirac, P. A. M. Quantised singularities in the electromagnetic field. Proc. Royal Soc. London. Ser. A, Containing Pap. a Math. Phys. Character 133, 60-72 (1931).

2. Polchinski, J. Monopoles, duality, and string theory. Int. J. Mod. Phys. A 19, 145-154 (2004).

3. Aad, G. et al. Search for magnetic monopoles and stable high-electric-charge objects in 13 tev proton-proton collisions with the atlas detector. Phys. review letters 124, 031802 (2020).

4. Fang, Z. et al. The anomalous hall effect and magnetic monopoles in momentum space. Science 302, 92-95 (2003).

5. Castelnovo, C., Moessner, R. \& Sondhi, S. L. Magnetic monopoles in spin ice. Nature 451, $42-45$ (2008).

6. Bramwell, S. T. et al. Measurement of the charge and current of magnetic monopoles in spin ice. Nature 461, 956-959 (2009).

7. Mól, L. et al. Magnetic monopole and string excitations in two-dimensional spin ice. J. Appl. Phys. 106, 063913 (2009).

8. Morgan, J. P., Stein, A., Langridge, S. \& Marrows, C. H. Thermal ground-state ordering and elementary excitations in artificial magnetic square ice. Nat. Phys. 7, 75-79 (2011).

9. Qi, Y., Brintlinger, T. \& Cumings, J. Direct observation of the ice rule in an artificial kagome spin ice. Phys. Rev. B 77, 094418 (2008).

10. Mól, L., Pereira, A. \& Moura-Melo, W. Extending spin ice concepts to another geometry: The artificial triangular spin ice. Phys. Rev. B 85, 184410 (2012).

11. Ribeiro, I. R. B. et al. Realization of rectangular artificial spin ice and direct observation of high energy topology. Sci. reports 7, 1-9 (2017).

12. Loreto, R. P. et al. Emergence and mobility of monopoles in a unidirectional arrangement of magnetic nanoislands. Nanotechnology 26, 295303 (2015).

13. Nisoli, C., Kapaklis, V. \& Schiffer, P. Deliberate exotic magnetism via frustration and topology. Nat. Phys. 13, 200-203 (2017).

14. Gilbert, I. et al. Emergent ice rule and magnetic charge screening from vertex frustration in artificial spin ice. Nat. Phys. 10, 670-675 (2014).

15. Macêdo, R., Macauley, G., Nascimento, F. \& Stamps, R. Apparent ferromagnetism in the pinwheel artificial spin ice. Phys. Rev. B 98, 014437 (2018).

16. Kapaklis, V. et al. Melting artificial spin ice. New J. Phys. 14, 035009 (2012). 
17. Silva, R., Nascimento, F., Mól, L., Moura-Melo, W. \& Pereira, A. Thermodynamics of elementary excitations in artificial magnetic square ice. New J. Phys. 14, 015008 (2012).

18. Wysin, G., Pereira, A., Moura-Melo, W. \& de Araujo, C. Order and thermalized dynamics in heisenberg-like square and kagome spin ices. J. Physics: Condens. Matter 27, 076004 (2015).

19. Silva, R. et al. Nambu monopoles interacting with lattice defects in a two-dimensional artificial square spin ice. Phys. Rev. $B$ 87, 014414 (2013).

20. Morley, S. A. et al. Thermally and field-driven mobility of emergent magnetic charges in square artificial spin ice. Sci. reports 9, 1-10 (2019).

21. Anghinolfi, L. et al. Thermodynamic phase transitions in a frustrated magnetic metamaterial. Nat. communications 6, 1-6 (2015).

22. Farhan, A. et al. Thermodynamics of emergent magnetic charge screening in artificial spin ice. Nat. communications $\mathbf{7}$, $1-6(2016)$.

23. Zhang, X. et al. Understanding thermal annealing of artificial spin ice. APL Mater. 7, 111112 (2019).

24. Nascimento, F., Mól, L., Moura-Melo, W. \& Pereira, A. From confinement to deconfinement of magnetic monopoles in artificial rectangular spin ices. New J. Phys. 14, 115019 (2012).

25. Loreto, R. et al. Experimental and theoretical evidences for the ice regime in planar artificial spin ices. J. Physics: Condens. Matter 31, 025301 (2018).

26. Gonçalves, R. et al. Tuning magnetic monopole population and mobility in unidirectional array of nanomagnets as a function of lattice parameters. Appl. Phys. Lett. 114, 142401 (2019).

27. Mól, L., Moura-Melo, W. \& Pereira, A. Conditions for free magnetic monopoles in nanoscale square arrays of dipolar spin ice. Phys. Rev. B 82, 054434 (2010).

28. Möller, G. \& Moessner, R. Artificial square ice and related dipolar nanoarrays. Phys. Rev. Lett. 96, 237202 (2006).

29. Perrin, Y., Canals, B. \& Rougemaille, N. Extensive degeneracy, coulomb phase and magnetic monopoles in artificial square ice. Nature 540, 410-413 (2016).

30. Farhan, A. et al. Emergent magnetic monopole dynamics in macroscopically degenerate artificial spin ice. Sci. advances $\mathbf{5}$, eaav6380 (2019).

31. Nisoli, C. Write it as you like it. Nat. nanotechnology 13, 5-6 (2018).

32. Arava, H. et al. Engineering relaxation pathways in building blocks of artificial spin ice for computation. Phys. Rev. Appl. 11, 054086 (2019).

33. Caravelli, F., Chern, G.-W. \& Nisoli, C. Artificial spin ice phase-change memory resistors. arXiv preprint arXiv:1908.08073 (2019).

34. Rodrigues, J. \& Mól, L. Towards magnetic monopole interaction measurement in artificial spin ice systems. J. Magn. Magn. Mater. 458, 327-334 (2018).

35. Kaur, M. et al. Magnetic reversal dynamics of nife-based artificial spin ice: Effect of nb layer in normal and superconducting state. J. Appl. Phys. 122, 193903 (2017).

36. Tersoff, J. \& Falicov, L. Magnetic and electronic properties of ni films, surfaces, and interfaces. Phys. Rev. B 26, 6186 (1982).

37. de Paiva, T. et al. Effects of magnetic monopoles charge on the cracking reversal processes in artificial square ices. Sci. reports 10, 1-7 (2020).

38. Moodera, J. \& Meservey, R. Magnetic proximity effect in thin films of ni on nonmagnetic metals. Phys. Rev. B 29, 2943 (1984).

39. Hong, S. C., Lee, J. I. \& Freeman, A. J. Proximity effects of overlayers on surface magnetism: Al adsorbed on fe (001). J. Magn. Magn. Mater. 99, L45-L54 (1991).

\section{Acknowledgements}

We would like to thank the agencies CNPq 432029/2018-1, FAPEMIG, Coordenação de Aperfeiçoamento de Pessoal de Nível Superior (CAPES) - Finance Code 001 and Newton Fund NAF292040 for the financial support. 


\section{Author contributions statement}

C.I.L.A. conceived the experiment(s), J.B. and P.P.F. developed the samples, T.S.P. performed aluminum cover, F.F.M. and D.G.D conducted the $M F M$ experiment(s), J.H.R and L.A.S.M carried simulations, L.A.S.M and C.I.L.A. analysed the results. All authors reviewed the manuscript.

\section{Additional information}

Competing interests The authors declare no competing interests. 


\section{Figures}
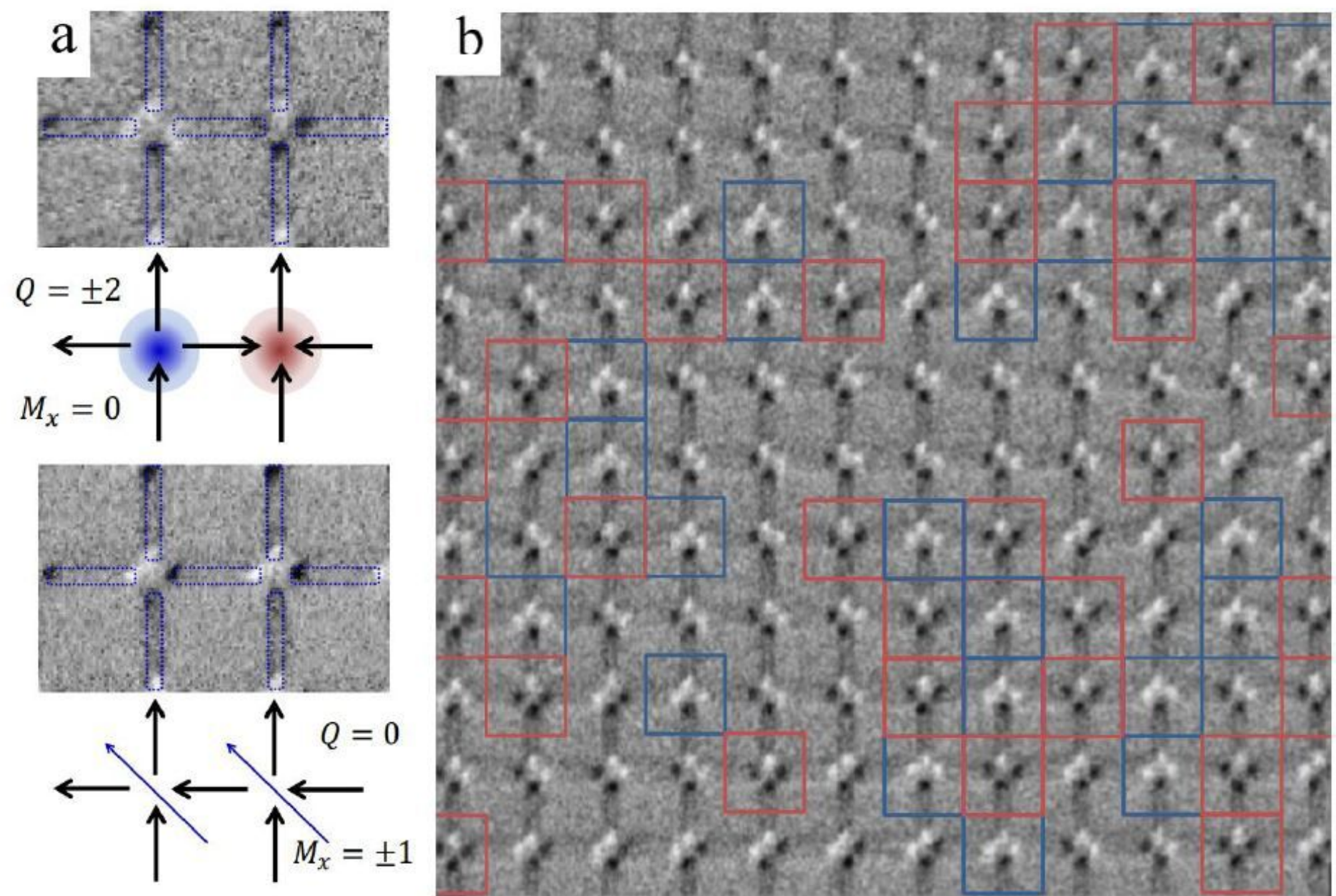

Figure 1

a) MFM measurement at vertex showing respectively 3 out - 1 in / 3 in - 1 out configurations allowing emergence of charges $Q=+2$ and $Q=-2$, followed by 2 in -2 out configuration of emergent dipole at vertex. b) Sample of measurement used to count magnetization and monopole density, realized during magnetization reversal process, with opposite charge monopoles highlighted by red and blue squares. 

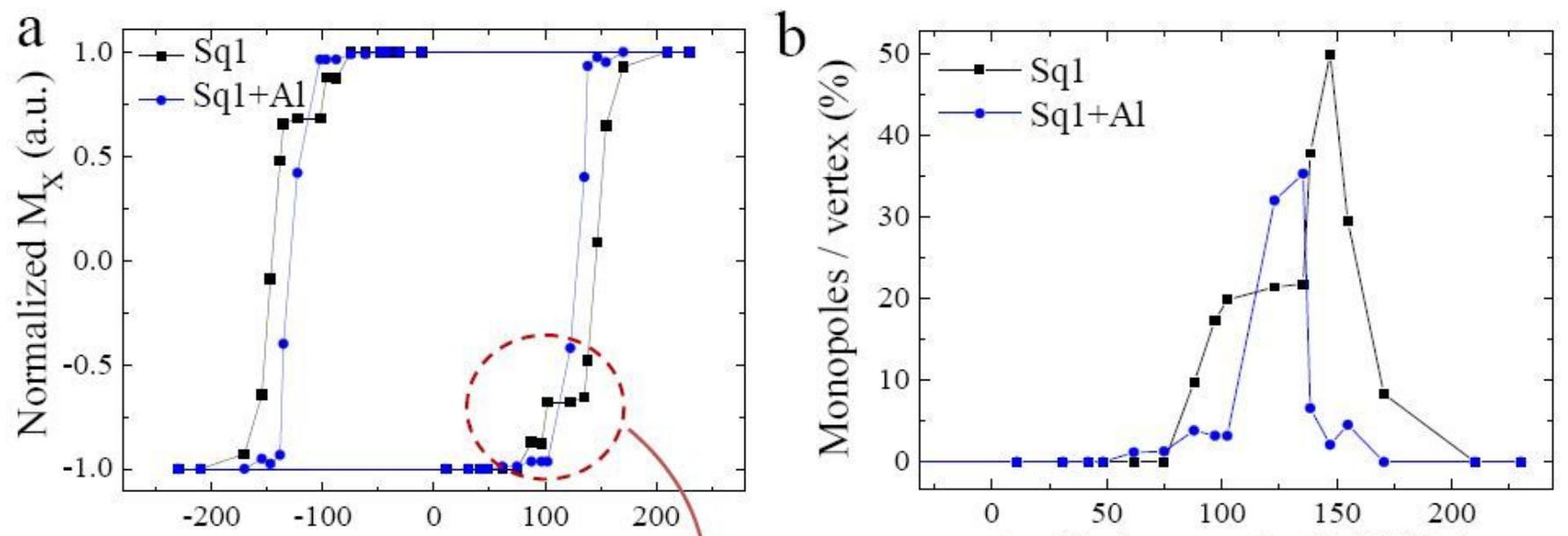

Applied magnetic field (Oe)

$\mathrm{c}$

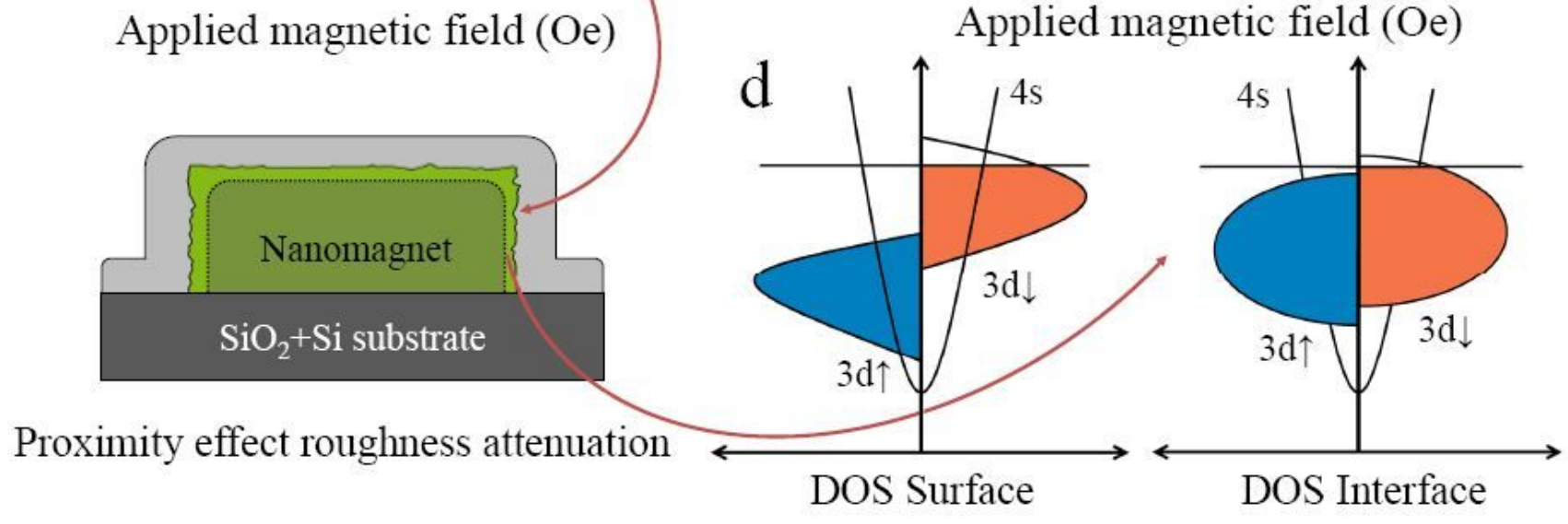

Figure 2

a) Hysteresis curve obtained by magnetization count at vertex during reversal process for Sq1 ASI sample and $\mathrm{ASI}$ samples covered by $20 \mathrm{~nm}$ aluminum Sq1+Al, b) Density of monopole evolution during reversal process, $c$ ) suggested defect smoothness by proximity effect and d) scheme of modification in density of state (DOS) by sp-d hybridization at the interface36. 

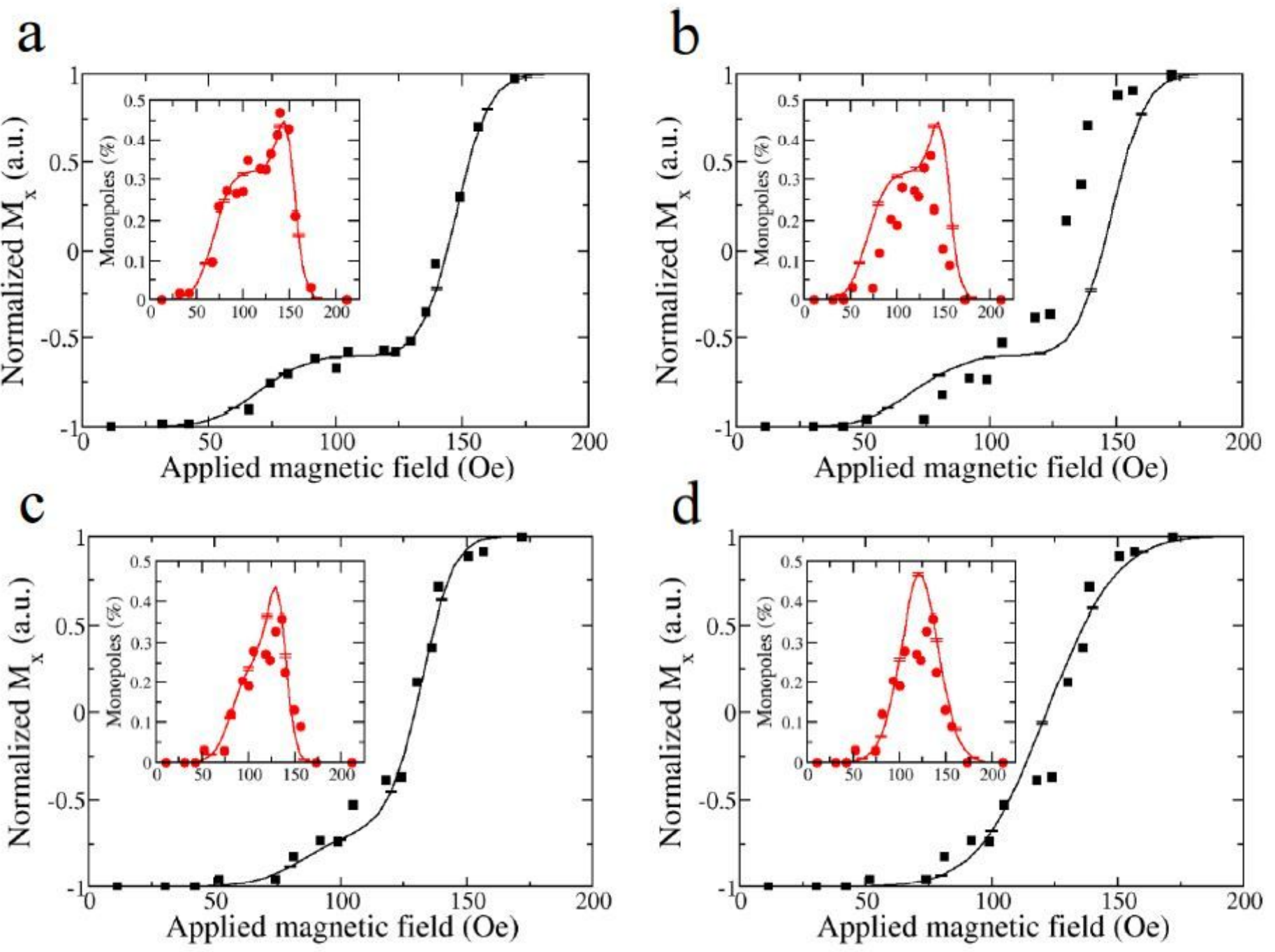

Figure 3

Please see the Manuscript PDF file for the complete figure caption. 